\title{
O cotidiano de trabalho do agente comunitário de saúde no PSF em Porto Alegre
}

\author{
Daily activities by community health workers \\ in the Family H ealth Program in Porto Alegre, Brazil
}

Lucimare Ferraz 1

Denise Rangel Ganzo de Castro Aerts 2

1 Centro de Ciências da Saúde, UNOChapecó. Av. Senador Atílio Fontana 591-E, Bloco G, 89809-000, Chapecó SC. Iferraz@unochapeco.edu.br 2 Programa de Pós-Graduação em Saúde Coletiva, ULBRA. Equipe de Informação, Coordenadoria Geral de Vigilância em Saúde, SM S-POA-RS.
Abstract This research aimed to study the daily work of the communitarian health agent $(\mathrm{CHA})$ in the Family $\mathrm{H}$ ealth Program (FHP) of Porto Alegre. The outline used was the series of cases, being the population under studies composed by $114 \mathrm{CH}$ As and $46 \mathrm{FH} \mathrm{P}$ professionals of the teams. The teams that had participated in this stage of the research had been selected in a stratified sampling of about $50 \%$ of the services of the FHP in each Sanitary District of Porto Alegre. The data collecting happened through half-structuralized questionnaires and the technique of the focal group. The main activity of the communitarian health agent is the home visit, mentioned by $2 / 3$ of the professionals. The health education is the second most developed one done by agents. Another activity of the agent is the support work to the teams, assisting in the reception of patients, handbook searches; phoning and organization and control of the warehouse. Despite of being the communitarian agent's main activity, the home visits weren't carried through with total effectiveness, once part of its time is dedicated to the administrative activities - not fitting, then, with its role. M oreover, the schedule designed for the visits is not adjusted to the local reality. The study shows strategies to maximize the agent's work potential in the FH P in Porto Alegre.

Key words Communitarian $\mathrm{H}$ ealth Agent, Family H ealth Program, Daily activities
Resumo Esta pesquisa teve como objetivo estudar o cotidiano de trabalho do agente comunitário de saúde (ACS) no Programa Saúde da Família (PSF) de Porto Alegre. 0 delineamento utilizado foi o de série de casos, sendo a população em estudo 114 ACS e 46 profissionais das equipes do PSF. As equipes que partici param dessa etapa da pesquisa foram selecionadas em uma amostragem estratificada de cerca de $50 \%$ dos serviços do PSF presentes em cada Distrito Sanitário de Porto Alegre. Para a obtenção dos dados foram utilizados questionários semi- estruturados e a técnica do grupo focal. A principal atividade do agente comunitário de saúde é a visita domiciliar, destacada por 2/3 dos profissionais. A educação em saúde éa segunda mais desenvolvida pelos agentes. O utra atividade do agente é o trabalho de apoio às equipes, auxiliando na recepção de paciente, busca de prontuários; telefonia e organização e controle do almoxarifado. A pesar de a visita domiciliar ser a principal atividade do agente comunitário, a pesquisa revela que esta não é realizada com total efetividade, uma vez que parte de seu tempo é dedicada a atividades administrativas o que descaracteriza sua função. Além disso, 0 horário destinado para as visitas não está adequado à realidade local. 0 estudo aponta estraté gias para potencializar o trabalho do agente no PSF em Porto Alegre.

Palavras-chave Agente Comunitário, Programa Saúde da Família, Cotidiano de trabalho 


\section{Introdução}

0 agente comunitário de saúde (ACS) é um trabal hador que atua em dois importantes programas do M inistério da Saúde: o Programa Agente Comunitário de Saúde (PACS) e o Programa Saúde da Família (PSF). Atualmente, esses programas consolidam-se no contexto da municipalização e descentralização das ações de atenção primária à saúde no Brasil.

Para o M inistério da Saúde, o agente comunitário de saúde é um trabalhador que faz parte da equipe de saúde da comunidade onde mora. É uma pessoa preparada para orientar famílias sobre cuidados com sua própria saúde e também com a saúde da comunidade (Brasil, 1999). Sem dúvida, esse trabalhador apresenta características especiais, uma vez que atua na mesma comunidade onde vive, tornando mais forte a relação entre trabal ho e vida social.

$\mathrm{N}$ a América Latina, a inserção dos agentes nos serviços de saúde ganhou impulso nos últimos anos, quando M inistérios da Saúde da América do Sul receberam apoio financeiro de agências internacionais para que começassem a capacitar e utilizar esses trabalhadores. Outro fator que contribuiu para sua inserção nos serviços de saúde foi que médicos e enfermeiros, além de serem profissionais caros para o sistema, não se dispunham a trabal har nas periferias e zonas rurais, sendo que um agente bem preparado poderia desenvolver cuidados básicos em saúde (Corrêa, 1995).

No Brasil, desde 1943, o M inistério da Saúde, através da Fundação de Serviço Especial de Saúde Pública (SESP), passou a formar pessoal auxiliar com o objetivo de ampliar as atividades das unidades de saúde para áreas desassistidas. Esses eram conhecidos como visitadores sanitários, guardas da malária e auxiliares de saneamento (Bastos, 1966).

$M$ ais recentemente, evidencia-se um aumento importante do número de agentes comunitários vinculados ao programa PSF que tem contribuído para uma profunda mudança no conceito de cuidados de saúde no País. Aproximadamente um quinto da população brasileira está em contato com as equipes de saúde do PSF (Unicef, 2001). Existem, atualmente, 17.608 equipes do Programa atuando em cerca de quatro mil municípios brasileiros, assistindo em torno de 57 milhões pessoas. A meta do governo federal é de que, até 0 ano de 2006, estejam atuando 32 mil equipes no atendimento de 100 milhões de pessoas, ou seja, cerca de 77\% da população brasileira (Brasil, 2004).

Considerando esse vertiginoso crescimento do Programa Saúde da Família, foi desenvolvida a presente pesquisa, com objetivo de investigar o cotidiano de trabalho do agente comunitário de saúde na cidade de Porto Alegre. Acredita-se ser importante conhecer como se processa seu universo de trabalho de forma a subsidiar políticas públicas de saúde e estratégias que potencializem as ações desse trabalhador, gerando um impacto positivo na saúde da população.

\section{Metodologia}

0 estudo foi desenvolvido em 29 unidades do PSF de Porto Alegre, no período entre julho e dezembro de 2000. 0 delineamento utilizado foi o de série de casos, composta por dois grupos: 1) todos os ACS do PSF, totalizando 114 sujeitos; e 2) três integrantes (um médico, um enfermeiro e um auxiliar de enfermagem) de cada uma das 16 equipes de saúde sorteadas, totalizando 46 sujeitos. Essas 16 equipes foram selecionadas por amostragem estratificada de cerca de $50 \%$ dos serviços do PSF presentes em cada um dos 11 Distritos Sanitários do município.

Para a obtenção dos dados, foram utilizados questionários semi-estruturados com perguntas abertas e fechadas. As entrevistas com os agentes foram realizadas nas unidades de saúde, onde também foram entregues os questionários auto-aplicáveis aos profissionais participantes do estudo.

Além dessa etapa individual de coleta dos dados, foi realizado um grupo focal, utilizando-se um roteiro com os temas sel ecionados. Fizeram parte do grupo focal 11 agentes selecionados, um moderador e um redator.

0 tratamento dos dados de natureza qualitativa foi feito por meio de Análise de Conteúdo Temática que permitiu a identificação das categorias. Para a análise quantitativa, foi utilizada a estatística descritiva com distribuição absoluta e relativa das respostas nas categorias investigadas.

$\mathrm{O}$ projeto de pesquisa foi submetido ao $\mathrm{Co}$ mitê de Ética em Pesquisa da Secretaria M unicipal de Saúde de Porto Alegre. Os participantes do estudo foram orientados em relação aos objetivos e procedimentos da pesquisa, tendo assinado o termo de consentimento livre e es- 
clarecido. Esta pesquisa atendeu a todas as orientações da Resolução 196/96 do Conselho Nacional de Saúde.

\section{Resultados}

O Programa Saúde da Família de Porto Alegre, no momento da coleta de dados, contava com 114 agentes comunitários de saúde, em sua mai oria mulheres, na faixa etária entre 30 e 49 anos (71\%), possuindo entre 9 e 11 anos de estudo, católicos, que viviam com companheiro, tinham um ou dois filhos e uma renda per capita entre 1,0 e 1,9 salários mínimos (Tabela 1). Em relação ao tempo de moradia na região, cerca de $73 \%$ eram moradores há mais de 10 anos na comunidade em que trabalham, sendo que o tempo de permanência no PSF também era alto, apresentando baixa rotatividade no programa: $64,8 \%$ trabal havam há mais de três anos e somente $8,8 \%$ não haviam completado um ano de trabalho (Tabela 2).

0 agente comunitário de saúde desenvolve várias atividades no Programa Saúde da Família em Porto Alegre (Tabela 3).

A principal atividade é a visita domiciliar, seguida da educação em saúde. De acordo com os agentes, nas atividades educativas, as orientações que mais costumam prestar às famílias referem-se à higiene; ao calendário vacinal; aos cuidados com recém-nascidos, puérperas, gestantes; e uso correto das medicações.

Outra atividade bastante destacada pelos profissionais das equipes foi o acompanhamento de idosos, crianças, gestantes, puérperas e grupos de risco. Alguns profissionais (21,7\%) relataram que os agentes também interagem com a equipe na formação de grupos dos programas de saúde. Para $19,6 \%$ dos profissionais das equipes de saúde, o agente participa no controle vacinal e no cadastramento das famílias da comunidade. A busca ativa de fal tosos acompanhados pelos programas também foi citada por $15,2 \%$ dos profissionais como atividade importante desenvolvida pelos agentes do PSF.

U m dado interessante é que, em $13 \%$ dos relatos feitos pela equipe, uma das atividades do agente em sua unidade é o trabalho de apoio à equipe, isso é, trabalho administrativo. Em contrapartida, no grupo focal essas atividades foram as mais discutidas.

Com relação à principal atividade do agente, a "visita domiciliar", investigaram-se al gu-

\section{Tabela 1}

Distribuição dos agentes comunitários de saúde segundo suas características sociodemográficas, Porto Alegre, RS, 2000.

\begin{tabular}{|c|c|c|}
\hline Variáveis & $\mathbf{n}$ & $\%$ \\
\hline \multicolumn{3}{|l|}{ Sexo } \\
\hline feminino & 101 & 88,6 \\
\hline masculino & 13 & 11,4 \\
\hline \multicolumn{3}{|l|}{ Faixa Etária (anos) } \\
\hline 19 a 29 & 26 & 22,8 \\
\hline 30 a 39 & 38 & 33,3 \\
\hline 40 a 49 & 43 & 37,7 \\
\hline 50 a 59 & 6 & 5,3 \\
\hline$>60$ & 1 & 0,9 \\
\hline \multicolumn{3}{|l|}{ Escolaridade (anos) } \\
\hline$<4$ & 4 & 3,5 \\
\hline 5 a 8 & 51 & 44,7 \\
\hline 9 a 11 & 57 & 50,0 \\
\hline$>12$ & 2 & 1,8 \\
\hline \multicolumn{3}{|l|}{ Religião } \\
\hline católica & 84 & 73,7 \\
\hline espírita & 11 & 9,6 \\
\hline evangélica & 6 & 5,3 \\
\hline protestante & 1 & 0,9 \\
\hline outras & 3 & 2,6 \\
\hline não tem religião & 9 & 7,9 \\
\hline \multicolumn{3}{|l|}{ Companheiro } \\
\hline $\operatorname{sim}$ & 76 & 66,7 \\
\hline não & 38 & 33,3 \\
\hline \multicolumn{3}{|l|}{ Filhos (número) } \\
\hline não tem & 15 & 13,1 \\
\hline 1 & 24 & 21,1 \\
\hline 2 & 33 & 28,9 \\
\hline 3 & 23 & 20,2 \\
\hline$>4$ & 19 & 16,7 \\
\hline \multicolumn{3}{|c|}{ Renda per capita (sm*) } \\
\hline$<1,0$ & 16 & 14,0 \\
\hline 1,0 a 1,9 & 53 & 46,5 \\
\hline 2,0 a 2,9 & 24 & 21,0 \\
\hline 3,0 a 3,9 & 13 & 11,4 \\
\hline 4,0 a 4,9 & 2 & 1,8 \\
\hline$>5,0$ & 6 & 5,3 \\
\hline Total & 114 & 100,0 \\
\hline
\end{tabular}

* Salário mínimo federal. 


\section{Tabela 2}

Distribuição dos agentes comunitários de saúde segundo tempo de trabalho no PSF e de moradia na comunidade, Porto Alegre, RS, 2000.

\begin{tabular}{lrr}
\hline Variáveis & $\mathbf{n}$ & $\%$ \\
\hline $\begin{array}{l}\text { Tempo de moradia } \\
\text { na comunidade (anos) }\end{array}$ & & \\
4 a 9 & 26 & 22,8 \\
10 a 19 & 44 & 38,6 \\
20 a 29 & 28 & 24,6 \\
30 a 39 & 11 & 9,6 \\
$>40$ & 5 & 4,4 \\
\hline Tempo de trabalho & & \\
no PSF (anos) & & \\
$\quad<$ de 1 & 10 & 8,8 \\
1 a 1,9 & 11 & 9,7 \\
2 a 2,9 & 19 & 16,7 \\
3 a 3,9 & 69 & 60,5 \\
$>4,0$ & 5 & 4,3 \\
\hline Total & $\mathbf{1 1 4}$ & $\mathbf{1 0 0 , 0}$ \\
\hline
\end{tabular}

\section{Tabela 3}

Distribuição das principais atividades desenvolvidas pelos agentes comunitários de saúde segundo a equipe de saúde do PSF, Porto Alegre, RS, 2000.

\begin{tabular}{lcc}
\hline Atividades Desenvolvidas & $\mathbf{n}$ & $\%$ \\
\hline visita domiciliar & 31 & 67,4 \\
educação em saúde & 15 & 32,6 \\
acompanhamento & 11 & 23,9 \\
de grupos de risco & & \\
incentivo e participação & 10 & 21,7 \\
na formação dos grupos & & \\
controle vacinal & 9 & 19,6 \\
cadastramento & 9 & 19,6 \\
busca ativa de faltosos & 7 & 15,2 \\
trabalho comunitário & 6 & 13,0 \\
controle e participação & 6 & 13,0 \\
nos programas de saúde & & \\
atividades burocráticas do posto & 6 & 13,0 \\
\hline
\end{tabular}

N esta tabela, a soma dos percentuais excede $100 \%$ em função dos Agentes Comunitários de Saúde terem mencionado mais de uma atividade. mas das características desta atividade (Tabela 4). 0 número de visitas domiciliares realizadas por dia é em média de sete a nove, sendo que 18,4\% ficaram abaixo da média recomendada pelo M inistério da Saúde, que é de no mínimo oito visitas diárias. $\mathrm{No}$ entanto, a maioria dos agentes tem um número elevado de famílias sob sua responsabilidade. Segundo os depoimentos dos agentes, o período do dia mais favorável para desenvolver essa atividade é o turno da tarde.

Foi também de interesse deste estudo investigar quais as atividades que 0 agente mais gosta e quais as que menos gosta de desenvolver em seu cotidiano. Entre as atividades mais apreciadas encontra-se a visita domiciliar, seguida por trabalhar com bebês e crianças. Os idosos também foram referidos como um grupo de pessoas com boa receptividade ao trabalho do agente em suas casas.

Em relação às atividades menos apreciadas, as atividades administrativas foram as mais destacadas. Essas consistem em atuar na recepção da unidade de saúde, realizar o agendamento de consultas, organizar pastas e prontuários, controlar materiais e almoxarifado. 0 preenchimento das fichas do Sistema de Informação de Atenção Básica (SIAB), que é uma atividade própria do agente, também foi considerado uma atividade desagradável.

\section{Discussão}

Todos os indivíduos selecionados aceitaram participar do estudo; não houve recusas. Com isso, foi possível que se conhecesse o cotidiano de trabalho dos agentes comunitários de saúde no Programa Saúde da Família em Porto Alegre.

A imensa maioria dos agentes entrevistados era composta por mulheres, assim como em outros municípios brasileiros ( $M$ artins et al., 1996). Essa tendência também é observada entre os profissionais da saúde, como enfermeiros, que é uma categoria profissional formada basicamente por mulheres (M achado, 2000). Esse fato pode estar intimamente ligado ao papel de cuidador que a mulher desempenha na sociedade, sendo as principais responsáveis pela educação e pela alimentação das crianças e pelos cuidados prestados aos membros idosos da família (Ellis et al., 1998).

Um dos pré-requisitos do Ministério da Saúde é que tenham idade acima de 18 anos, não 
sendo estabelecido um limite máximo (Brasil, 2001b). A faixa etária que mais concentrou agentes foi entre 30 e 49, isto é, adultos jovens.

Acredita-se que os agentes comunitários de saúde com mais idade tendem a conhecer meIhor a comunidade, ter mais vínculos e laços de amizades, porém podem ter algumas inimizades ou conflitos com outros moradores. Eles também têm seus próprios conceitos sobre 0 processo saúde-doença, advindos de experiências próprias ou al heias, podendo ser mais resistentes a novos conceitos relacionados à promoção da saúde em sua comunidade. Por outro lado, os agentes mais jovens não conhecem tão bem a comunidade, seu envolvimento pode ser menor; entretanto, poderão não ter inimizades, seus conceitos de saúde e doença poderão não ser muito arraigados, estando mais abertos às mudanças e às novidades.

Em outros estudos (Andrade, 1998; Silva \& Dalmaso, 2002), os autores também encontraram uma concentração de indivíduos entre 30 a 45 anos, sendo que Silva e Dalmaso (2002) destacam que, para muitos, ser agente comunitário de saúde foi uma oportunidade de reingresso no mercado de trabal ho eque, para outros, ser agente representou reconhecimento e remuneração do trabalho já desenvolvido como voluntários na comunidade.

Em novembro de 1996, quando foram implantadas as primeiras oito equipes do PSF em Porto Alegre, esse trabalho ainda era uma novidade para a comunidade, sendo que os primeiros a se candidatarem para o cargo foram pessoas com mais idade. Segundo o relato de alguns, eles foram convidados para participar das seleções pelos presidentes da associação de moradores e líderes comunitários, pois já desenvolviam atividades em prol da comunidade.

A situação etária encontrada já deve, possivelmente, ter sofrido algumas mudanças, pois se percebe que os agentes que ingressaram mais recentemente no Programa eram os mais jovens.

0 crescimento dos programas em que 0 agente está inserido abre em todo o País oportunidades de emprego às populações mais carentes, tendo em vista que a implantação do programa tem como prioridade as comunidades de baixa renda. Como não exige grau de escolaridade, todos que sabem ler e escrever podem candidatar-se ao processo seletivo, que acaba selecionando os "mais qualificados" para tal função. Sabe-se que nessas comunidades os mais jovens geralmente têm maior escolaridade, sendo melhores classificados.

\section{Tabela 4}

Distribuição dos agentes comunitários de saú de segundo variáveis referentes à prática de visitas domiciliares (VD) no PSF, Porto Alegre, RS, 2000.

\begin{tabular}{lll}
\hline Variáveis & $\mathbf{n}$ & $\%$ \\
\hline $\begin{array}{l}\text { Período do dia mais } \\
\text { favorável para VD }\end{array}$ & \\
manhã & 15 & 13,1 \\
tarde & 72 & 63,2 \\
ambos os turnos & 27 & 23,7
\end{tabular}

\begin{tabular}{lrr}
\hline $\begin{array}{l}\text { Média de VD por } \\
\text { dia de trabalho }\end{array}$ & \\
4 a 6 & 21 & 18,4 \\
7 a 9 & 67 & 58,8 \\
10 a 12 & 20 & 17,5 \\
14 a 16 & 6 & 5,3 \\
\hline Total & 114 & 100,0 \\
\hline
\end{tabular}

\section{Número de famílias}

por ACS

120 a $169 \quad 12 \quad 10,6$

170 a $219 * \quad 33 \quad 29,2$

220 a $269 \quad 32 \quad 28,3$

270 a $319 \quad 22 \quad 19,5$

320 a $369 \quad 8 \quad 7,1$

$>370 \quad 6 \quad 5,3$

$\begin{array}{lll}\text { Total } & 113^{* *} & 100,0\end{array}$

* Faixa na qual se inclui a média de famílias por ACS preconizada pelo M inistério da Saúde.

** U ma ACS não informou esses dados porque era nova no programa e o cadastramento das suas famílias ainda não estava concluído.

Os critérios de seleção variam para cada município, no entanto, a realização de entrevistas permite verificar o grau de conhecimento do candidato, assim como identificar os que apresentam um perfil mais adequado para 0 trabal ho com as comunidades. Para que isso de fato ocorra, esse método de seleção deve ser bem elaborado e desenvolvido por pessoas competentes para tal função, possibilitando a seleção de candidatos mais aptos. Infelizmente, ainda em alguns municípios brasileiros, a contratação desses trabalhadores é feita por escolha pessoal ou partidária (Corbo et al., 2000).

Em Porto Alegre, todos os agentes comunitários passaram por um processo seletivo, 0 que acaba se refletindo no nível de escolaridade, pois a maioria apresentava o ensino fundamental completo. O M inistério da Saúde não exige grau de escolaridade para a função de agente, somente que saiba ler e escrever (Brasil, 1999). No entanto, quanto maior o grau de es- 
colaridade mais condições terá o agente de incorporar novos conhecimentos e orientar as famílias sob sua responsabilidade.

É importante conhecer a religião dos ACS, pois as crenças pessoais podem influenciar em sua relação com a comunidade e no papel que devem desempenhar. Além disso, é sabido que o estado de saúde é determinado também por valores culturais e religiosos (Waldman, 1999). Para alguns, a doença é considerada uma punição de Deus ou vinda de um mal feito por espíritos. Muitos aceitam a doença como uma vontade divina ou destino (H elman, 1994). É por essa razão que a forma como as pessoas promovem ou preservam sua saúde está intimamente ligada ao modo como acreditam adquirir as doenças (Waldman, 1999). Assim, ressalta-se a importância de capacitar os profissionais para o reconhecimento dos fatores culturais e religiosos que possam influenciar no comportamento dos indivíduos com relação à sua saúde. É certo que isso não significa para o trabalhador da saúde abdicar de suas crenças e valores, mas sim a possibilidade de uma meIhor articulação entre os saberes técnicos e populares.

Tendo em vista que o agente vai assistir pessoas de outras crenças, é imprescindível prepará-lo para lidar com as questões religiosas da comunidade, inclusive com a sua, para que possa tratar com habilidade de assuntos como os que estão ligados à sexualidade, por exemplo.

Quanto à família, dois terços dos agentes viviam com companheiro(a), sendo que o restante morava sozinho, com amigos ou parentes. A grande maioria desses trabal hadores já havia passado pelo exercício da maternidade ou paternidade, trazendo consigo vivências sobre saúde infantil geradas por suas experiências no cuidado de seus filhos. Se por um lado essas experiências podem facilitar o trabaIho junto das famílias; por outro, o que deu certo em suas experiências pessoais pode não ser 0 adequado para a população por eles assistida. Mais uma vez, torna-se fundamental a educação permanente dos agentes e a supervisão constante desse trabalhador pela equipe de saúde.

A renda per capita dos agentes caracterizaos como população de baixa renda, tendo uma inserção econômica muito semelhante a da população por eles assistida. Esse resultado mostra também que muitos deles têm um papel importante na sustentação econômica de suas famílias, uma vez que têm como remuneração por seu trabalho um salário que correspondia na época da pesquisa a dois salários mínimos federais.

Segundo o M inistério da Saúde, um agente comunitário deve ganhar pelo menos um salário mínimo por mês. Os recursos para efetuar o pagamento do seu salário provêm em parte do governo federal, do governo municipal e, em menor proporção, do governo estadual (Brasil, 2001b). Outro ponto importante em relação aos trabalhadores do PSF é que, nesse programa, os recursos humanos não são contratados como funcionários públicos e podem ser remunerados por uma combinação de procedimentos e produtividade, isto é, remunerados por desempenho (Viana et al., 1998).

A maioria dos agentes morava na mesma comunidade em que trabalhava por um período entre 10 a 19 anos, sendo que o tempo mínimo relatado foi de quatro anos. Preenchem, portanto, o pré-requisito do M inistério da Saúde que estabelece que 0 agente deve estar morando, no mínimo, há dois anos no local onde trabal hará, ressaltando que são os únicos trabalhadores da atenção primária à saúde que devem obrigatoriamente residir na área de atuação (Brasil, 1994a).

Esse tempo de dois anos é solicitado como uma condi ção necessária para que 0 agente conheça a comunidade. Por outro lado, é importante considerar que a qualidade da relação entre agente e comunidade é apenas em parte determinada pelo tempo de sua residência no local, pois a sua empatia com a comunidade e vice-versa são fatores importantes para a integração desse trabalhador.

Quanto ao tempo de trabalho no PSF, encontrou-se uma baixa rotatividade desses trabal hadores, sendo que al guns dos que estão há menos tempo iniciaram suas atividades em serviços recém implantados. 0 tempo de permanência no Programa é importante para 0 entendimento do papel do agente, que é construído nas suas práticas cotidianas.

A visita domiciliar éuma das principais atividades preconizadas pelo M inistério da Saúde para o agente comunitário (Brasil, 2001b) e também foi a mais referida no presente estudo. É por meio dela que o agente melhor conhece as necessidades das famílias e, principalmente, desenvolve o trabal ho educativo, citado como a segunda atividade mais realizada.

A maioria dos agentes comunitários de saúde, quando perguntados nas entrevistas individuais sobre qual era o período do dia de maior 
rendimento para as visitas domiciliares, respondeu ser o período da tarde. Segundo eles, na parte da manhã é mais difícil a realização das visitas, pois as pessoas dormem atétarde e as donas de casa estão mais ocupadas com os afazeres domésticos, não dispondo de muito tempo para atendê-los. Sobre isso, um agente referiu: "saímos pra rua às 9-9:30h e eles ainda estão dormindo".

Constata-se, entretanto, uma divergência entre o período mais favorável para a realização das visitas domiciliares (tarde) e o período em que os agentes estão desenvolvendo tal atividade (manhã). No turno da tarde, a maior parte do tempo é utilizada em atividades dentro da unidade de saúde. Com isso, está ocorrendo uma perda da eficiência dessas visitas.

A visita domiciliar é o instrumento ideal para a educação em saúde, pois a troca de informações se dá no contexto de vida do indivíduo e de sua família. As orientações não estão prontas, pois cada casa apresenta uma realidade e é baseada nessa realidade que acontece a troca de informações.

Segundo o relato de um dos agentes, muitas vezes as pessoas não percebem seus problemas. No entanto, durante uma visita domiciliar é possível observar as condições do pátio, da casa e iniciar uma conversa de "comadre" sobre questões de saúde. Nessas ocasiões, 0 agente tem a oportunidade de identificar problemas e orientar, "trocar idéias", no mesmo "papo" sobre cuidados em saúde. Como relatou um agente durante a entrevista: não basta só bater na porta e perguntar se tem um problema de saúde, tem que sentar e conversar.

0 agente é responsável por 750 pessoas de sua comunidade, sendo esse número flexível, pois depende das necessidades locais, e deve visitar cada domicílio pelo menos uma vez por mês (Brasil, 2001b). No entanto, para a maioria dos agentes, não é possível visitar todas as famílias mensalmente, pois o número é muito elevado. Essa situação foi evidenciada no presente estudo, o que determina, segundo os agentes entrevistados, priorizarem as famílias que necessitam de um maior acompanhamento, como aquelas com pessoas em tratamento médico, com tuberculose, AIDS, doença mental, gestantes, puérperas e crianças em risco nutricional.

Além do excesso do número de famílias, o número de horas dispensadas para essa atividade éinsuficiente e há a inadequação já referida do horário em que realizam as visitas. As- sim, considerando essas dificuldades, 0 agente age coerentemente ao selecionar as famílias a serem visitadas, garantindo que as que mais necessitem não fiquem sem acompanhamento adequado.

Evidencia-se, assim, a necessidade de readequação da atividade de visitas domiciliares, de acordo com as características da população de cada local, assim como o resgate junto às equipes de saúde do significado e da importância dessa prática para o Programa. É possível que, com a carga de doenças e de necessidades desses grupos populacionais, o número de famílias sob responsabilidade de cada equipe extrapole sua capacidade de resposta, necessitando talvez de um redimensionamento do número de profissionais que atuam em cada unidade do PSF.

Com relação ao trabalho educativo prestado pelo agente, M artins e colaboradores (1996) ressaltam que a participação desse em ações pontuais e simplificadas parece produzir impacto significativo e relevante em termos de Saúde Coletiva, como, por exemplo, a orientação de uso do soro caseiro para a redução da mortalidade infantil e a cloração na água para a prevenção da cólera.

Outra atividade do agente em sua unidade é $o$ trabalho de apoio à equipe, referido como "trabalho burocrático". Esse tipo de trabalho, apesar de ser citado em uma freqüência relativamente baixa pelos profissionais das equipes (13\%), foi intensamente relatado e discutido pel os agentes, ficando claro que desenvolvem, além das atividades inerentes a sua função, 0 apoio administrativo às equipes do PSF.

0 trabal ho de apoio a que se referem são atividades como atender na recepção, entregando fichas para consultas médicas; procurar prontuários de pacientes; atender ao telefone; entregar medicação na farmácia e marcar consultas especializadas para a comunidade, por solicitação médica, na Central de $M$ arcação de Consultas na SM S. Segundo relatos de alguns agentes, a contabilidade da farmácia e o pedido de materiais para a Secretaria M unicipal de Saúde também eram tarefas suas.

Constatamos que na maioria dos serviços do PSF em Porto Alegre existe uma rotina preestabelecida em relação ao trabal ho do agente na unidade de saúde. Em algumas, há uma escala diária ou semanal do agente que ficará na unidade para auxiliar a equipe. Outros serviços não possuem uma escala fixa, sendo solicitado conforme as necessidades diárias. 
Os agentes referiram que permanecem boa parte do seu tempo, na maioria das vezes no período da tarde, auxiliando a equipe de saúde em trabal hos burocráticos. Vale lembrar que o tempo que 0 agente permanece nas unidades de saúde diminui sua disponibilidade para as visitas. Outra ressalva é de que em alguns casos as visitas podem estar sendo realizadas muito rapidamente, comprometen do a qualidade da atenção à população assistida.

0 trabalho de suporte que 0 agente vem prestando às equipes de saúde sinaliza que, além de ser sobrecarregado com tarefas de sua atribuição, passa a ser um "tapa-buraco" da carência de outros profissionais nos serviços de saúde (Vasconcel os, 1997). Sobre isso, um agente externou o que segue: Diz que chamaram o agente lá em Tramandaí (cidade do litoral do RS) de bombril: mil e uma utilidades.

Cabe aqui lembrar que 0 agente comunitário de saúde tem como função identificar problemas, orientar, encaminhar e acompanhar a realização dos procedimentos necessários à proteção, à promoção, à recuperação e à reabilitação da saúde dos moradores de cada casa sob sua responsabilidade (Brasil, 2001b). 0 estudo mostrou que 0 agente vem desenvolvendo al gumas atividades que descaracterizam o seu papel, uma vez que não estão preconizadas pelo M inistério da Saúde.

Diante dos resultados obtidos, sugere-se algumas ações que talvez possam potencializar 0 trabalho do ACS como, incorporar um auxiliar administrativo nas equipes do PSF; diminuir o número de famílias por ACS; e oferecer mais capacitações e educação permanente no Programa.

Por fim, é importante ressaltar que, apesar de os agentes atenderem às equipes nos aspectos práticos do funcionamento dos serviços de saúde, eles vêm também desenvolvendo importante trabal ho na vigilância da saúde da população.

0 agente é uma peça importante para 0 desenvolvimento do PSF, assim sendo, cuidar desse trabalhador e valorizá- lo é de fundamental importância, pois "aqueles que atuam e promovem saúde, ou seja, trabalhadores, são um bem público, uma utilidade pública" (M achado, 1995).

\section{Colaboradores}

LF trabalhou no delineamento do estudo, coleta, análise e interpretação dos dados, na redação do artigo e revisão da versão final. DRGCA trabalhou no delineamento do estudo, análise e interpretação dos resultados, redação do artigo e revisão da versão final. 


\section{Referências bibliográficas}

Andrade FM O 1998. 0 Programa de Saúde da Família no Ceará. Dissertação de mestrado em saúde pública pela Universidade Federal do Ceará. Fortaleza.

Brasil 1994. Programa de A gentes Comunitários de Saúde: N ormas e Diretrizes. M inistério da Saúde, Brasília/ DF.

Bastos NC 1966. Treinamento de pessoal de saúde pública: 23 anos de experiência da Fundação Serviço Especial de Saúde Pública 1942-1965. Rio de Janeiro: Estado da Guanabara.

Brasil 1999. Saúde da família no Brasil: linhas estratégicas para o quadriênio 1999/2002. M inistério da Saúde, Brasília.

Brasil 2001a. Programa Saúde da Família - PSF. Secretaria Executiva, M inistério da Saúde, Brasília.

Brasil 2001b. Programa Agentes Comunitários de SaúdePACS. Secretaria Executiva, M inistério da Saúde, Brasília.

Brasil 2004. Programa Saúde da Família - PSF. M inistério da Saúde. Disponível em <http://dtr2001.saude.gov.br/ psf/equipes/quantos.asp>. Acessado em 16/07/2004.

Corbo AA et al. 2000. M apeamento das experiências de PACS/PSF em curso no Estado do Rio de Janeiro. Rio de Janeiro (M imeo).

Corrêa ME 1995. Um pouco de história, pp. 37-45. In M inistério da Saúde. 0 trabalho do Agente Comunitário de Saúde. Brasília.

Ellis JR \& H artley CL 1998. Enfermagem contemporânea: desafios, questões e tendências. Trad. de M aria Virgínia Godoy da Silva. (5a ed.). Artmed, Porto Alegre.

H elman CG 1994. Cultura, saúde e doença. (2a ed.). Artes M édicas, Porto Alegre.
M achado MH (coord.) 2000. Perfil dos médicos e enfermeiros do Programa Saúde da Família no Brasil. Ministério da Saúde, Brasília.

$M$ artins CL et al. 1996. Agentes comunitários nos serviços de saúde pública: elementos para uma discussão. Revista Saúde D ebate 51:38-43.

M inayo M CS 1993. 0 desafio do conhecimento: pesquisa qualitativa em saúde. (2a ed.). Hucitec-Abrasco, São Paulo-Rio de Janeiro.

Silva JÁ \& Dalmaso ASW 2002. Agente Comunitário de Saúde: o ser, o saber, o fazer. Fiocruz, Rio de Janeiro.

U nicef - Fundo das N ações Unidas para a Infância. Relatório Situação da Infância Brasileira 2001. D esenvolvimento infantil - os primeiros seis anos de vida.

Vasconcelos E 1997. Os novos profissionais: as auxiliares de saúde, pp. 51-57. In E Vasconcelos. Educação popular nos serviços de saúde. (3ạ ed.). Hucitec, São Paulo.

Viana AL \& Dal Poz M 1998. A reforma do sistema de saúde no Brasil e o Programa de Saúde da Família. Reforma, tecnologia e recursos humanos. Physis Revista de Saúde Coletiva 8(2):11-48.

Waldman BF 1999. 0 enfoque cultural no processo de educação em saúde. Dissertação de mestrado. Rede de Pós-Graduação em Enfermagem da Região Sul Repensul, Porto Alegre.

Artigo apresentado em 5/10/2004

Aprovado em 8/12/2004

Versão final apresentada em 8/12/2004 